\title{
Perihippocampal metastasis following hippocampus-avoiding prophylactic cranial irradiation for small cell lung cancer: a case report
}

This article was published in the following Dove Press journal:

OncoTargets and Therapy

II August 2017

Number of times this article has been viewed

\section{Seung-Gu Yeo}

Department of Radiation Oncology, Soonchunhyang University College of Medicine, Soonchunhyang University Hospital, Cheonan, Republic of Korea
Correspondence: Seung-Gu Yeo Department of Radiation Oncology, Soonchunhyang University College of Medicine, Soonchunhyang University Hospital, 3I, Soonchunhyang 6-gil, Cheonan 3II5I, Republic of Korea Tel +82 4I 5703557 Fax +82 4I 5923809 Email md6630@schmc.ac.kr
Abstract: Prophylactic cranial irradiation (PCI) lowers the risk of brain metastasis (BM) and increases survival in small cell lung cancer (SCLC) patients, but it also entails a risk of neurocognitive dysfunction (NCD). One strategy to mitigate this neurotoxicity is hippocampusavoiding (HA) whole-brain radiation therapy, as the hippocampus is mainly responsible for radiation-related NCD and hippocampal or perihippocampal metastases are rare. A few prospective clinical trials have demonstrated a reduction in NCD following HA whole-brain radiation therapy. The 59-year-old male patient described in this report had limited-stage SCLC and a complete response to thoracic chemoradiotherapy. Seven months after receiving HA-PCI of $25 \mathrm{~Gy}$ in 10 fractions using intensity-modulated radiation therapy, a $36 \mathrm{~mm}$ solitary metastasis was detected in the right perihippocampal region. The mass was surgically removed but the patient died 2 months later. The development of a solitary HA region metastasis is uncommon, considering that metastasis in this area usually occurs in patients with high numbers of BMs. Our case demonstrates the need for further validation of HA-PCI for SCLC patients in terms of both neurocognitive protection and the absence of compromise in terms of BM prevention.

Keywords: hippocampus-sparing, lung cancer, neurotoxicity, cognition, brain metastasis, whole brain radiation therapy

\section{Introduction}

Prophylactic cranial irradiation (PCI) is the standard of care for patients with small cell lung cancer (SCLC) who have had a good response to chemoradiation for their primary disease..$^{1}$ In a meta-analysis, PCI was shown to reduce the incidence of brain metastasis (BM) from $58 \%$ to $33 \%$ and to improve overall survival by $5.4 \%$ at 3 years. ${ }^{2}$ However, a side effect of PCI is neurocognitive dysfunction (NCD). In the clinical trials of the Radiation Therapy Oncology Group (RTOG), PCI increased the risk of NCD in lung cancer patients, who did not develop BM, by almost threefold. ${ }^{3}$ Common manifestations of NCD include decreased short-term memory, impaired cognition, fatigue, and depression., ${ }^{4,5}$

Injury to the hippocampus is mainly responsible for NCD following whole-brain radiation therapy (WBRT). ${ }^{6}$ Preclinical and clinical evidence have shown a correlation between hippocampal radiation injury and NCD. ${ }^{7,8}$ Therefore, one strategy to mitigate NCD is to reduce the irradiation dose to the hippocampus during WBRT. Modern, sophisticated radiation therapy (RT) techniques allow the delivery of hippocampusavoiding (HA) WBRT, in both the treatment of BM and during PCI. ${ }^{9,10}$ However, the risk of subsequent metastasis in this conformal avoidance region must be evaluated to determine the clinical feasibility of HA-WBRT. 
In this article, we report the outcome of a patient with limited-stage SCLC who received HA-PCI but subsequently developed a solitary BM in the hippocampal avoidance region.

\section{Case report}

A 59-year-old male was admitted to the hospital due to blood-tinged sputum. He was a current smoker with a 30-year smoking history. Chest computed tomography (CT) revealed an $18 \mathrm{~mm}$ mass in the right upper lobe with enlarged right prevascular, upper paratracheal, hilar, interlobar, and bilateral lower paratracheal lymph nodes. Distant metastasis was not detected on an ${ }^{18} \mathrm{~F}$-fluorodeoxyglucose positron emission tomography $\left({ }^{18} \mathrm{~F}-\mathrm{FDG}-\mathrm{PET}\right) / \mathrm{CT}$, whole-body bone scan or on magnetic resonance imaging (MRI) of the brain. Endobronchial ultrasound-guided biopsy identified metastatic small cell carcinoma bilaterally in the lower paratracheal nodes. Written informed consent was provided by the patient to have the case details and any accompanying images published.

Definitive concurrent chemoradiotherapy was administered for this limited-stage SCLC, with a total of 64 Gy delivered in 32 fractions using static seven-field intensitymodulated radiotherapy (IMRT). Chemotherapy, consisting of cisplatin and etoposide, was commenced simultaneously with IMRT. Four cycles of chemotherapy were administered over a 3-week interval. Chest CT and ${ }^{18} \mathrm{~F}$-FDG-PET/CT performed 2 months after chemoradiation showed a complete response in both the primary lung tumor and the regional lymph nodes.

HA-PCI was performed 3 months after completion of thoracic chemoradiotherapy. The IMRT techniques followed the RTOG 0933 protocol, ${ }^{11}$ a Phase II trial of HA-WBRT for patients with BM. For CT (Brilliance CT Big Bore; Philips
Medical Systems, Cleveland, OH, USA) simulation, the patient was immobilized in the supine position with an aquaplast mask placed over his head. Planning CT scan images were fused with axial brain MRI. The hippocampus was contoured bilaterally on the fused MRI-CT image set and expanded by $5 \mathrm{~mm}$ to generate the HA region. The planning target volume was defined as the whole-brain parenchyma, excluding the HA region. The volumes of the hippocampus, $\mathrm{HA}$ region, and whole-brain parenchyma were $5.1 \mathrm{~cm}^{3}$, $30.4 \mathrm{~cm}^{3}$, and $1,343.8 \mathrm{~cm}^{3}$, respectively. The percentage of brain volume occupied by the HA region was $2.3 \%$. An IMRT plan was created using the Eclipse RT planning system (Varian Medical Systems, Inc., Palo Alto, CA, USA). The plan consisted of nine static fields to cover the planning target volume, with a dose of 25 Gy administered in 10 fractions while avoiding the hippocampus (Figure 1). The minimum, maximum, and mean doses to the hippocampus were $7.6 \mathrm{~Gy}$, 13.6 Gy, and 8.7 Gy, respectively. IMRT was performed using a Novalis Tx system (Varian Medical Systems and BrainLab, Feldkirchen, Germany). The patient tolerated the treatments well, with no severe complications.

Brain MRI at 7 months after HA-PCI completion showed a $36 \times 31 \mathrm{~mm}$, well-marginated, heterogeneously enhancing mass in the right HA (perihippocampal) region with surrounding edema (Figure 2). The patient's symptoms included lower extremity weakness and dysarthria. The mass was surgically removed and identified by pathology as metastatic small cell carcinoma. The patient subsequently received palliative RT for spinal metastasis, and chemotherapy, but he died 9 months after HA-PCI.

\section{Discussion}

The efficacy of HA-WBRT in reducing NCD has been assessed in prospective clinical trials, ${ }^{11,12}$ including
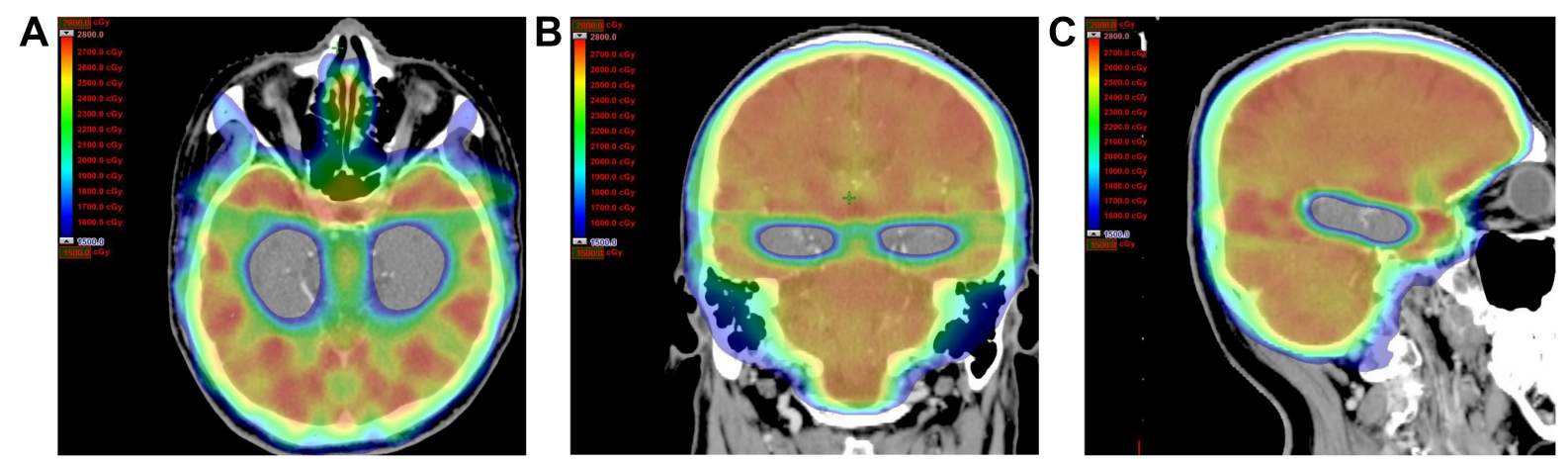

Figure I Radiotherapy planning image with the dose distribution illustrated in color.

Notes: (A) Axial, (B) coronal, and (C) sagittal images. The planning target volume was the whole-brain parenchyma excluding HA region.

Abbreviation: HA, hippocampus-avoiding. 

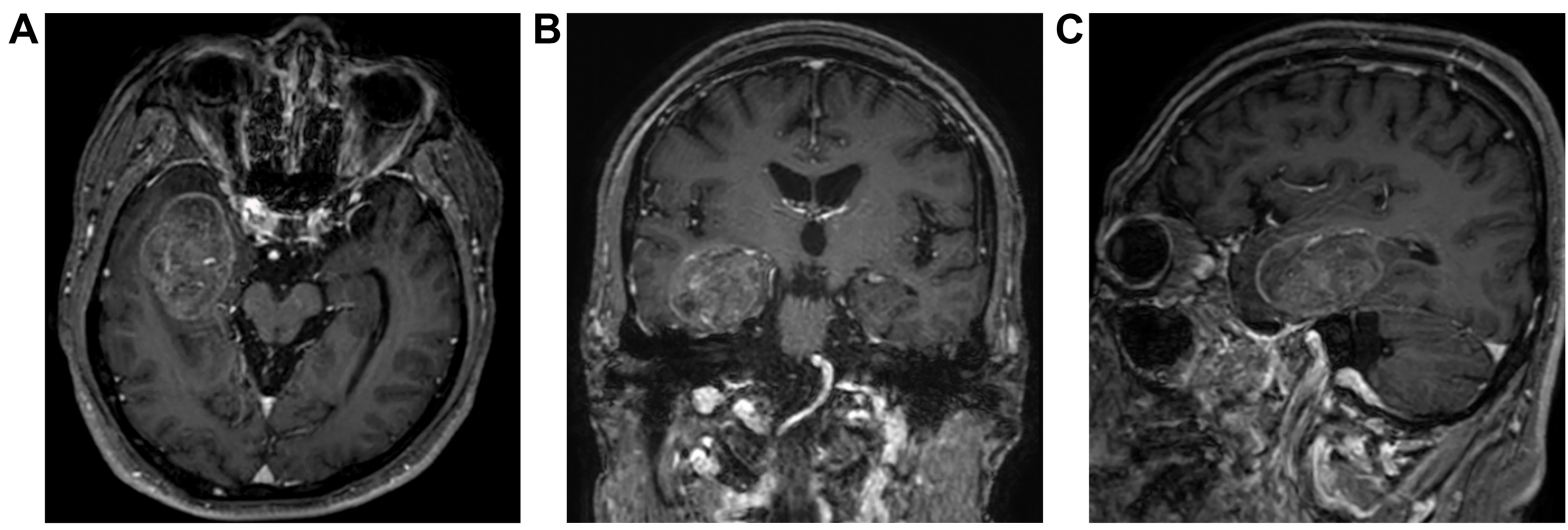

Figure 2 Brain magnetic resonance images showing a 36×31 mm metastatic mass in the right perihippocampal area. Note: (A) Axial, (B) coronal, and (C) sagittal images.

RTOG 0933, a Phase II multi-institutional study of HAWBRT (30 Gy in 10 fractions) for patients with BM. ${ }^{11}$ The trial included patients with BMs located outside a $5 \mathrm{~mm}$ margin around the hippocampus, with a total of 100 patients analyzed. The mean relative decline in delayed recall from baseline to 4 months, as assessed on the Hopkins Verbal Learning Test - Revised, was significantly lower than that in the historical control ( $7 \%$ vs $30 \%, P<0.001)$; also, there was no decline in quality-of-life scores. Of the 67 patients who developed intracranial progression, only three $(4.5 \%)$ experienced progression in the HA area. However, the patients included in the study mostly had non-small cell lung cancer (NSCLC) and breast cancer; those with SCLC were excluded from that clinical trial.

Although sample size was small (20 patients), a recent prospective study demonstrated the potential benefit of HAPCI for SCLC patients in limiting the neuropsychological sequelae of brain radiation. ${ }^{13}$ Two patients developed a metastasis in a region under-dosed during HA-PCI. Neither involved the hippocampal dentate gyrus, but one involved the HA region. Both patients developed additional concurrent metastases in the fully treated brain regions. In the current case, BM was detected in the HA region 7 months after IMRT-based HA-PCI. A distinctive feature of this case was that the BM was solitary. No concurrent BM outside the HA region was evident, and no other BM was shown prior to death.

One of the rationales for the use of HA-WBRT is that the hippocampus and perihippocampal area are uncommon sites of BM. Two retrospective studies examined the incidence of hippocampal metastasis exclusively in SCLC patients. ${ }^{14,15}$ Guo et a ${ }^{14}$ assessed a total of 1,594 BMs (at presentation or follow-up) in 180 SCLC patients. HA region metastasis was detected in $22(12.2 \%)$ patients: 13 perihippocampal ( $\leq 5 \mathrm{~mm}$ of hippocampus) and nine hippocampal metastases. Kundapur et a $1^{15}$ evaluated 70 SCLC patients with BM either at presentation (de novo) or after conventional WBRT for BM. Of the 59 patients with de novo BM, three (5\%) had HA region metastasis: two hippocampal and one perihippocampal. Collectively, there were 359 (range: 1-33) de novo BMs, with only three $(0.8 \%)$ within $\leq 5 \mathrm{~mm}$ of hippocampal tissue. In 20 patients, metastatic disease in the brain progressed after WBRT, but a perihippocampal metastasis developed in only one patient (5\%).

Other retrospective studies have analyzed BM in patients whose primary tumors were mainly NSCLC, breast cancer, and melanoma, but also SCLC. BM in the HA region was detected in $<10 \%$ of the patients. ${ }^{16-20}$ Gondi et al ${ }^{16}$ determined a rate of $8.6 \%(n=32)$ in 371 patients with BM. This rate was not significantly different between patients with $\operatorname{SCLC}(10.5 \% ; \mathrm{n}=38)$ and NSCLC $(9.0 \% ; \mathrm{n}=155)$. However, a higher rate in SCLC than in other malignancies has also been reported. In a study by Harth et al, ${ }^{17} \mathrm{BM}$ centered within $5 \mathrm{~mm}$ around the hippocampus occurred in $27.3 \%$ of SCLC patients. Although there were only 11 SCLC patients, this rate contrasted with that of $8 \%$ in overall 100 patients. Wan et $\mathrm{al}^{18}$ demonstrated that $\mathrm{BM}$ in regions of neural stem cells accounted for $2.7 \%$ of all BMs in 44 SCLC patients, compared with $0.82 \%$ of all BMs in the 283 NSCLC patients.

HA-WBRT will be of greater relevance for prophylactic than for therapeutic purposes. In BM treatment, particularly in brain oligometastases, stereotactic radiosurgery omitting WBRT is gaining acceptance. ${ }^{21,22}$ Stereotactic radiosurgery targets only the metastatic tumor volume, sparing all of the noninvolved brain parenchyma. Most of the ongoing prospective clinical trials of HA-WBRT in lung cancer patients are 
evaluating the efficacy of HA-PCI rather than therapeutic WBRT. ${ }^{6}$ In addition, patients with NSCLC may be more suitable HA-PCI candidates versus patients with SCLC, as the former have a lower tendency of BM occurrence within the HA area. ${ }^{17,18}$ However, PCI has not been shown to confer a survival benefit in NSCLC. ${ }^{23,24}$

A known risk factor for HA region metastasis is a large number of BMs. ${ }^{14,18}$ The current case was therefore unusual as this patient had a single BM in the HA region. Although this solitary BM was removed by surgical resection, stereotactic radiosurgery may be a good alternative. ${ }^{25}$

While PCI is accepted as the standard of care for patients with lung cancer, it is used suboptimally because of neurotoxicity concerns. ${ }^{26}$ The rapid development of RT technology has enabled the realization of HA-PCI. This novel approach will find widespread acceptance following its rigorous validation in prospective clinical trials to examine its ability to confer neurocognitive protection and, importantly, to retain the efficacy of BM prevention.

\section{Acknowledgment}

This work was supported by the Soonchunhyang University Research Fund.

\section{Disclosure}

The author reports no conflicts of interest in this work.

\section{References}

1. Pechoux CL, Sun A, Slotman BJ, De Ruysscher D, Belderbos J, Gore EM. Prophylactic cranial irradiation for patients with lung cancer. Lancet Oncol. 2016;17(7):e277-e293.

2. Auperin A, Arriagada R, Pignon JP, et al. Prophylactic cranial irradiation for patients with small-cell lung cancer in complete remission. Prophylactic cranial irradiation overview collaborative group. $N$ Engl J Med. 1999;341(7):476-484.

3. Gondi V, Paulus R, Bruner DW, et al. Decline in tested and self-reported cognitive functioning after prophylactic cranial irradiation for lung cancer: pooled secondary analysis of Radiation Therapy Oncology Group randomized trials 0212 and 0214. Int J Radiat Oncol Biol Phys. 2013;86(4):656-664.

4. Sun A, Bae K, Gore EM, et al. Phase III trial of prophylactic cranial irradiation compared with observation in patients with locally advanced non-small-cell lung cancer: neurocognitive and quality-of-life analysis. J Clin Oncol. 2011;29(3):279-286.

5. Qu XM, Mishra MV, Bauman GS, et al. Cost-effectiveness of prophylactic cranial irradiation with hippocampal avoidance in limited stage small cell lung cancer. Radiother Oncol. 2017;122(3):411-415.

6. Zhao R, Kong W, Shang J, Zhe H, Wang YY. Hippocampal-sparing whole-brain radiotherapy for lung cancer. Clin Lung Cancer. 2017;18(2): 127-131.

7. Pospisil P, Kazda T, Hynkova L, et al. Post-WBRT cognitive impairment and hippocampal neuronal depletion measured by in vivo metabolic MR spectroscopy: results of prospective investigational study. Radiother Oncol. 2017;122(3):373-379.

8. Son Y, Yang M, Wang H, Moon C. Hippocampal dysfunctions caused by cranial irradiation: a review of the experimental evidence. Brain Behav Immun. 2015;45:287-296.
9. Lee K, Lenards N, Holson J. Whole-brain hippocampal sparing radiation therapy: volume-modulated arc therapy vs intensity-modulated radiation therapy case study. Med Dosim. 2016;41(1):15-21.

10. Rong Y, Evans J, Xu-Welliver M, et al. Dosimetric evaluation of intensity-modulated radiotherapy, volumetric modulated arc therapy, and helical tomotherapy for hippocampal-avoidance whole brain radiotherapy. PLoS One. 2015;10(4):e0126222.

11. Gondi V, Pugh SL, Tome WA, et al. Preservation of memory with conformal avoidance of the hippocampal neural stem-cell compartment during whole-brain radiotherapy for brain metastases (RTOG 0933): a phase II multi-institutional trial. J Clin Oncol. 2014;32(34):3810-3816.

12. Lin SY, Yang CC, Wu YM, et al. Evaluating the impact of hippocampal sparing during whole brain radiotherapy on neurocognitive functions: a preliminary report of a prospective phase II study. Biomed $J$. 2015;38(5):439-449.

13. Redmond KJ, Hales RK, Anderson-Keightly H, et al. Prospective study of hippocampal-sparing prophylactic cranial irradiation in limitedstage small cell lung cancer. Int J Radiat Oncol Biol Phys. 2017;98(3): 603-611.

14. Guo WL, He ZY, Chen Y, et al. Clinical features of brain metastases in small cell lung cancer: an implication for hippocampal sparing whole brain radiation therapy. Transl Oncol. 2017;10(1):54-58.

15. Kundapur V, Ellchuk T, Ahmed S, Gondi V. Risk of hippocampal metastases in small cell lung cancer patients at presentation and after cranial irradiation: a safety profile study for hippocampal sparing during prophylactic or therapeutic cranial irradiation. Int J Radiat Oncol Biol Phys. 2015;91(4):781-786.

16. Gondi V, Tome WA, Marsh J, et al. Estimated risk of perihippocampal disease progression after hippocampal avoidance during whole-brain radiotherapy: safety profile for RTOG 0933 . Radiother Oncol. 2010; 95(3):327-331.

17. Harth S, Abo-Madyan Y, Zheng L, et al. Estimation of intracranial failure risk following hippocampal-sparing whole brain radiotherapy. Radiother Oncol. 2013;109(1):152-158.

18. Wan JF, Zhang SJ, Wang L, Zhao KL. Implications for preserving neural stem cells in whole brain radiotherapy and prophylactic cranial irradiation: a review of 2270 metastases in 488 patients. $J$ Radiat Res. 2013;54(2):285-291.

19. Wu SG, Rao MY, Zhou J, et al. Distribution of metastatic disease in the brain in relation to the hippocampus: a retrospective single-center analysis of 6064 metastases in 632 patients. Oncotarget. 2015;6(41): 44030-44036.

20. Ghia A, Tome WA, Thomas S, et al. Distribution of brain metastases in relation to the hippocampus: implications for neurocognitive functional preservation. Int J Radiat Oncol Biol Phys. 2007;68(4):971-977.

21. Brown PD, Jaeckle K, Ballman KV, et al. Effect of radiosurgery alone vs radiosurgery with whole brain radiation therapy on cognitive function in patients with 1 to 3 brain metastases: a randomized clinical trial. JAMA. 2016;316(4):401-409.

22. Kocher M, Soffietti R, Abacioglu U, et al. Adjuvant whole-brain radiotherapy versus observation after radiosurgery or surgical resection of one to three cerebral metastases: results of the EORTC 22952-26001 study. J Clin Oncol. 2011;29(2):134-141.

23. Gore EM, Bae K, Wong SJ, et al. Phase III comparison of prophylactic cranial irradiation versus observation in patients with locally advanced non-small-cell lung cancer: primary analysis of radiation therapy oncology group study RTOG 0214. J Clin Oncol. 2011;29(3): 272-278.

24. Park HS, Decker RH, Wilson LD, Yu JB. Prophylactic cranial irradiation for patients with locally advanced non-small-cell lung cancer at high risk for brain metastases. Clin Lung Cancer. 2015;16(4): 292-297.

25. Won YK, Lee JY, Kang YN, et al. Stereotactic radiosurgery for brain metastasis in non-small cell lung cancer. Radiat Oncol J. 2015;33(3): 207-216.

26. Giuliani M, Sun A, Bezjak A, et al. Utilization of prophylactic cranial irradiation in patients with limited stage small cell lung carcinoma. Cancer. 2010;116(24):5694-5699. 
OncoTargets and Therapy

\section{Publish your work in this journal}

OncoTargets and Therapy is an international, peer-reviewed, open access journal focusing on the pathological basis of all cancers, potential targets for therapy and treatment protocols employed to improve the management of cancer patients. The journal also focuses on the impact of management programs and new therapeutic agents and protocols on

patient perspectives such as quality of life, adherence and satisfaction. The manuscript management system is completely online and includes a very quick and fair peer-review system, which is all easy to use. Visit http://www.dovepress.com/testimonials.php to read real quotes from published authors.

Submit your manuscript here: http://www.dovepress.com/oncotargets-and-therapy-journal 